\title{
Glassy behavior of light
}

\author{
L. Angelani ${ }^{1}$, C. Conti $^{2,3}$, G. Ruocco ${ }^{3,4}$, F. Zamponi ${ }^{3,4}$ \\ ${ }^{1}$ Research center SMC INFM-CNR, c/o Università di Roma "La Sapienza," I-00185, Roma, Italy \\ ${ }^{2}$ Centro studi e ricerche "Enrico Fermi," Via Panisperna 89/A, I-00184, Roma, Italy \\ ${ }^{3}$ Research center Soft INFM-CNR, c/o Università di Roma "La Sapienza," I-00185, Roma, Italy \\ ${ }^{4}$ Dipartimento di Fisica, Università di Roma "La Sapienza," I-00185, Roma, Italy
}

\begin{abstract}
We study the nonlinear dynamics of a multi-mode random laser using the methods of statistical physics of disordered systems. A replica-symmetry breaking phase transition is predicted as a function of the pump intensity. We thus show that light propagating in a random non-linear medium displays glassy behavior, i.e. the photon gas has a multitude of metastable states and a non vanishing complexity, corresponding to mode-locking processes in random lasers. The present work reveals the existence of new physical phenomena, and demonstrates how nonlinear optics and random lasers can be a benchmark for the modern theory of complex systems and glasses.
\end{abstract}

PACS numbers: $64.70 . P f, 75.10 . \mathrm{Nr}, 42.55 . \mathrm{Zz}$

The first marriage between statistical mechanics and lasers dates back to their early development [1, 2]. Since the seventies, many authors have outlined that the threshold for lasing can be interpreted as a thermodynamic phase transition; and these ideas spread out in the field of photonics, later embracing also nonlinear optics (for a review see e.g. [3]). In recent articles, [4, 5, 6, 7, 8] the statistical properties of laser light in homogeneous cavities has been studied taking into account nonlinear phenomena, like gain saturation and intensity dependent refractive index. This nonlinearity gives rise to an interaction among the oscillation modes, which in turn produces new interesting effects. Specifically, by mapping the dynamics in an ordered Hamiltonian problem, the authors of Ref. 8] predicted a critical behavior (a phase transition) of the laser mode-locking process.

In the latter example, the statistical mechanics of ordered systems is applied to study light propagation in amplifying homogeneous non-linear materials. In recent years, the locally inhomogeneous character of matter, and in particular the disordered nature of these inhomogeneities, are becoming more and more important. Specifically, relevant attention has been dedicated to light amplification in random media and random lasers 9, 10, 11, 12, 13, 14]. This is a fascinating topic that bridges various fields like light localization and diffusion, thermodynamics, nonlinear physics and quantum optics, and it has relevant fundamental and applicative perspectives, as in bio-physics [15]. Methods of statistical mechanics have not yet been applied to the propagation of light in non-linear disordered active media, but the rich behavior observed in glasses (aging, memory effects, etc.) can be foreseen [16, 17].

In this Letter, we analytically study the statistical properties of the modes of a random optical cavity. Various physical settings are embraced by this problem: for example a micro-structured cavity filled by an active softmaterial, like doped liquid crystals, cavity-less random lasers, or even a standard laser system with a disordered amplifying medium. In all these cases, the disorder varies on time scales much longer than the optical fields (it is "quenched" [16]) and, for any realization of the system, the supported electromagnetic modes interact because of the nonlinearity of the resonant medium.

We first show that light propagation is described by an Hamiltonian with the pumping rate acting as inverse temperature. The mode amplitudes are taken as slowly varying and their phases play the role of "spins" in an Hamiltonian, which turns out to be a generalization of the XY model [18] and of the so called $k$-trigonometric model 19]. The thermodynamics of the model is studied using the replica trick [16], and a one step replica symmetry breaking transition is found [20]. Our results show that when the average energy into each mode increases (i.e. the "temperature" is decreased), the system undergoes a glass transition, meaning that its dynamics slows down and an exponentially large number of states appears, with a non vanishing "complexity" [20]. They correspond to "mode-locked" states of a random laser. This treatment somehow generalizes to disordered systems early works on multi-mode lasers (see e.g. 21, 22, 23]), and can be also applied to other problems involving multi-mode interactions in a nonlinear medium. By providing an analytically treatable statistical model, not only we unveil the complex behavior (in the meaning of modern glassy physics) of light in active disordered media, but we also demonstrate the possibility of using them for testing the replica symmetry breaking transitions. When studying the glass transition of atomic or molecular systems, due to the predicted kinetic arrest, the interesting time scale becomes extremely long with respect to that experimentally accessible. Due to the intrinsically fast photon dynamics, it is reasonable to expect that the system can be equilibrated much closer to the transition, and this should provide new and interesting experimental data on kinetically arrested "photon glasses".

We consider a dielectric resonator described by a refractive index profile $n(\mathbf{r})$. The time dependence of this quantity is of no interest here, taking place on a time scale much slower than that of the photon propagation. Our approach follows the standard coupled mode-theory 
in the time domain [24]. The Maxwell's equations in the presence of a nonlinear polarization $\mathbf{P}_{N L}$ are:

$$
\begin{aligned}
& \nabla \times \mathbf{H}(\mathbf{r}, t)=\varepsilon_{0} n^{2}(r) \partial_{t} \mathbf{E}(\mathbf{r}, t)+\partial_{t} \mathbf{P}_{N L}(\mathbf{r}, t, \mathbf{E}, \mathbf{H}) \\
& \nabla \times \mathbf{E}(\mathbf{r}, t)=-\mu_{0} \partial_{t} \mathbf{H}(\mathbf{r}, t) .
\end{aligned}
$$

The fields $\mathbf{E}(\mathbf{r}, t)$ and $\mathbf{H}(\mathbf{r}, t)$ are conveniently decomposed in normal modes corresponding to the solutions of the linear problem, $\mathbf{P}_{N L}=0$, (frequencies $\omega_{m}$, eigenvectors $\mathbf{E}_{m}(\mathbf{r}), \mathbf{H}_{m}(\mathbf{r})$, and "amplitudes" $\left.a_{m}\right)$. For later convenience, they are cast in the form

$$
\begin{aligned}
& \mathbf{E}(\mathbf{r}, t)=\sqrt{\omega_{m}} \Re\left[\sum_{m} a_{m} \mathbf{E}_{m}(\mathbf{r}) \exp \left(-i \omega_{m} t\right)\right], \\
& \mathbf{H}(\mathbf{r}, t)=\sqrt{\omega_{m}} \Re\left[\sum_{m} a_{m} \mathbf{H}_{m}(\mathbf{r}) \exp \left(-i \omega_{m} t\right)\right],
\end{aligned}
$$

where $\mathbf{E}_{m}(\mathbf{r}), \mathbf{H}_{m}(\mathbf{r})$ solve the eigenvalue problem

$$
\left(\begin{array}{cc}
0 & i \nabla \times \\
-i \nabla \times & 0
\end{array}\right)\left(\begin{array}{c}
\mathbf{E}_{m} \\
\mathbf{H}_{m}
\end{array}\right)=\omega_{m}\left(\begin{array}{cc}
\epsilon_{0} n^{2} & 0 \\
0 & \mu_{0}
\end{array}\right)\left(\begin{array}{l}
\mathbf{E}_{m} \\
\mathbf{H}_{m}
\end{array}\right)
$$

and are normalized such that

$$
\frac{1}{4} \int_{V}\left\{\varepsilon_{0} n^{2}(r)\left|\mathbf{E}_{m}(\mathbf{r})\right|^{2}+\mu_{0}\left|\mathbf{H}_{m}(\mathbf{r})\right|^{2}\right\} d V=1,
$$

where $V$ is the standard normalization volume, over which periodical boundary conditions are enforced. With these notations, the total energy stored in the cavity is $\mathcal{E}=\Sigma_{m} \mathcal{E}_{m}=\Sigma_{m} \omega_{m}\left|a_{m}\right|^{2}$.

In the presence of nonlinearity, the amplitudes $a_{m}$ are time-dependent and their evolution is described by coupled equations that can be derived using standard perturbation techniques 24] and take the general form

$$
\frac{d a_{m}(t)}{d t}=i \frac{\sqrt{\omega_{m}}}{4} \int_{V} \mathbf{E}_{m}^{*}(\mathbf{r}) \cdot \mathbf{P}_{m}(\mathbf{r}) d V .
$$

The integral in Eq. (4) is extended to the cavity volume where the nonlinear polarization is different from zero. The quantity $\mathbf{P}_{m}(\mathbf{r})$ is the amplitude of the component of the nonlinear polarization oscillating at $\omega_{m}$, $\mathbf{P}_{N L}=\Re\left[\sum_{m} \mathbf{P}_{m} \exp \left(-i \omega_{m} t\right)\right]$ and its explicit expression as function of the fields depends on the considered non-linearity. For isotropic media the leading cubic terms are written, in our notation, as 25]

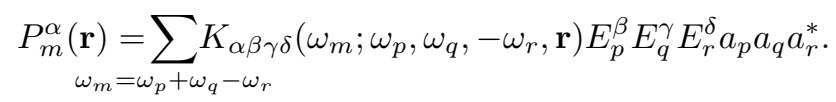

Here $K_{\alpha \beta \gamma \delta}=\sqrt{\omega_{p} \omega_{q} \omega_{r}} \chi_{\alpha \beta \gamma \delta}$ and $\chi$ is the third order response susceptibility tensor (explicit expressions are known for example in the two level approximation 22, 25, 26]); the sum over Cartesian indeces is implicit. The coupled mode theory equations in a nonlinear cavity read hence as

$$
\frac{d a_{m}(t)}{d t}=-\frac{1}{2} \Sigma_{p q r} g_{m p q r} a_{p} a_{q} a_{r}^{*},
$$

where the sum is extended over all the modes and

$$
g_{m p q r}=\frac{\sqrt{\omega_{m}}}{2 i} \int_{V} K_{\alpha \beta \gamma \delta}\left(\omega_{m} ; \omega_{p}, \omega_{q},-\omega_{r}, \mathbf{r}\right) E_{m}^{\alpha} E_{p}^{\beta} E_{q}^{\gamma} E_{r}^{\delta} d V .
$$

Under standard approximations [22, 25], the tensor $g$ is a quantity symmetric with respect to the exchange of any couple of indeces and can be taken as real-valued, neglecting processes like self and cross-phase modulations between modes (S-XPM). These mechanisms can be included in the model (see below), but will be here neglected for the sake of simplicity. Introducing the (real valued) function

$$
H=\frac{1}{4} \Sigma_{\text {spqr }} g_{\text {spqr }} a_{s} a_{p} a_{q}^{*} a_{r}^{*},
$$

and taking into account radiation losses and material absorption processes, represented by the coefficients $\alpha_{m}$ and light amplification through $\gamma_{m}$ (see also [24]), the resulting equation of motion for $a_{m}(t)$ is written as:

$$
\frac{d a_{m}}{d t}=-\frac{\partial H}{\partial a_{m}^{*}}+\left(\gamma_{m}-\alpha_{m}\right) a_{m}+\eta_{m}(t)=-\frac{\partial \mathcal{H}}{\partial a_{m}^{*}}+\eta_{m}(t),
$$

where $\mathcal{H}=\sum_{m}\left(\alpha_{m}-\gamma_{m}\right)\left|a_{m}\right|^{2}+H$, and having introduced as usual the noise term (see e.g. 2, 27]), with $\left\langle\eta_{p}(t) \eta_{q}\left(t^{\prime}\right)\right\rangle=2 k_{B} T_{b a t h} \delta_{p q} \delta\left(t-t^{\prime}\right)$, weighted by an effecting temperature $T_{b a t h}$, with $k_{B}$ the Boltzmann constant. The previous equation is a standard Langevin model for a system of $N$ particles moving in $2 N$ dimensions (represented by $\left.\left\{\Re a_{m}, \Im a_{m}\right\}_{m=1 . . N}\right)[2,[6]$ and its invariant measure is given by $\exp \left(-\mathcal{H} / k_{B} T_{\text {bath }}\right)$. Note that the latter is not affected by S-XPM terms, as we will discuss in future publications and originally addressed in $[6]$.

We consider a large number of modes in a small frequency interval $\omega_{m} \sim \omega_{0}$ pumped and put into oscillations. We can write $a_{m}(t)=A_{m}(t) \exp \left[i \varphi_{m}(t)\right]$, which is useful as we expect $A_{m}$ to be slowly varying with respect to $\varphi_{m}$, as discussed below. In previous works, with reference to standard multi-mode lasers, 21, 22 the phasedependent terms in (8), were always averaged out by assuming the $\varphi_{m}$ rapidly varying, independent and uniformly distributed. The resulting equations determine the oscillation energy $\mathcal{E}_{m}$ into each mode. However, for an increasing number of modes, nonlinear beatings induce non-trivial light dynamics which is mainly due to the rapidly varying phases, while the amplitudes can still be taken as slowly varying [21, 24, 28].

Summing up, the Hamiltonian depending on the relevant dynamic variables, the phases $\varphi_{m}$, is written as

$$
\mathcal{H}(G, \varphi)=\mathcal{H}_{o}+\Sigma_{s p q r} G_{\text {spqr }} \cos \left(\varphi_{s}+\varphi_{p}-\varphi_{q}-\varphi_{r}\right)
$$

where $\mathcal{H}_{o}=\sum_{m}\left(\alpha_{m}-\gamma_{m}\right) A_{m}^{2}$ is an irrelevant constant term and $G_{s p q r}=g_{s p q r} A_{s} A_{p} A_{q} A_{r}$. Hereafter we will consider these $G$ coefficients as "quenched" (due to the slow $t$ dependence of $A_{m}$ ), and the relevant phase space is reduced to that spanned by $\varphi_{m}$.

If the cavity is realized by a random medium, as described above, the coupling coefficients $G$ are random variables. Their values depend on the mode profiles, the resonant frequencies, and on the quenched values of the energies $\mathcal{E}_{m}=\omega_{m} A_{m}^{2}$ in each mode, which vary with 
each realization of the cavity for a given pumping rate. For these reasons we take $G_{s p q r}$ as random Gaussian variables. Due to the fact that the mode frequencies are typically symmetrically distributed with respect to the transition frequency of the amplifying atomic system (and correspondingly the signs of the nonlinear susceptibility terms largely varies, see e.g. [25, 26]) and their spread is small, $\omega_{m} \sim \omega_{o}, G$ is taken with zero mean value $\langle G\rangle=0$. The latter hypothesis can be removed by a suitable, but not trivial, generalization of the treatment below 16 .

The $G$ s roughly scale as $\left\langle A^{2}\right\rangle^{2} V^{-3 / 2}$, as one can derive from Eq. (6) by using that $E_{m}^{\alpha}$ is $O\left(V^{-1 / 2}\right)$ and $K_{\alpha \beta \gamma \delta}$ is a random variable integrated over $V$. Recalling that $\omega_{m} \sim \omega_{0}, \omega_{0}\left\langle A^{2}\right\rangle$ measures the average energy per mode. By a simple rescaling, the invariant measure can be written as $\exp [-\beta H(J, \varphi)]$, where $J_{s p q r}=G_{s p q r} /\left(g_{0}\left\langle A^{2}\right\rangle^{2}\right)$ has standard deviation $\propto 1 / V^{3 / 2} \propto 1 / N^{3 / 2}$ and $g_{0}$ is a material-dependent constant. Note that this scaling of the $J_{\mathrm{s}}$ guarantees that the Hamiltonian is extensive [16, 20]. The parameter that controls the phase transition is then $\beta \equiv 1 / T \equiv\left\langle A^{2}\right\rangle^{2} g_{0} / k_{B} T_{\text {bath }}$. Thus, in what follows transitions obtained as $\beta$ is increased (i.e. the adimensional effective temperature $T$ is decreased), can be controlled by increasing the amount of energy stored on average into each mode (i.e. the pumping rate).

The calculation of the thermodynamics goes through the evaluation of the partition function

$$
Z(J)=\int d \varphi e^{-\beta H(J, \varphi)},
$$

and, more specifically, of the free energy $f(\beta)$ averaged over the quenched disorder, that can be written, using the replica trick [16], as

$$
-\beta f(\beta)=\lim _{N \rightarrow \infty} \frac{1}{N} \overline{\log Z(J)}=\lim _{N \rightarrow \infty} \lim _{n \rightarrow 0} \frac{\overline{Z^{n}(J)}-1}{n N} .
$$

By a standard saddle-point evaluation, the replicated partition function is $\overline{Z^{n}(J)}=\exp [-N n \min g(q)]$, where the overlap matrix $q$ is defined by $q_{a b}=$ $N^{-1} \sum_{j} e^{i\left(\varphi_{j}^{a}-\varphi_{j}^{b}\right)}$ and one has to find the overlap values $\bar{q}$ that satisfy the saddle-point equation. The free energy is then given by

$$
\beta f=-\lim _{n \rightarrow 0}(n N)^{-1}\left\{e^{-N n g(\bar{q})}-1\right\} \sim \lim _{n \rightarrow 0} g(\bar{q})=\beta \phi(\bar{q}),
$$

where

$$
\begin{aligned}
& \beta \phi(q)=-\frac{\beta^{2}}{4}+\frac{3 \beta^{2}}{2} n^{-1} \Sigma_{a>b}\left|q_{a b}\right|^{4}-n^{-1} \log Z(q), \\
& Z(q)=\int d \varphi^{a} \exp \left[\Re \Sigma_{a>b} 2 \beta^{2}\left|q_{a b}\right|^{2} q_{a b}^{*} e^{i\left(\varphi^{a}-\varphi^{b}\right)}\right] .
\end{aligned}
$$

This function is very similar to the one that describes the Ising $p$-spin glass for $p=4$, see e.g. [20]. The derivative with respect to $q_{a b}$ of $f(q)$ gives the saddle point equation

$$
q_{a b}=\left\langle\exp \left[i\left(\varphi^{a}-\varphi^{b}\right)\right]\right\rangle
$$

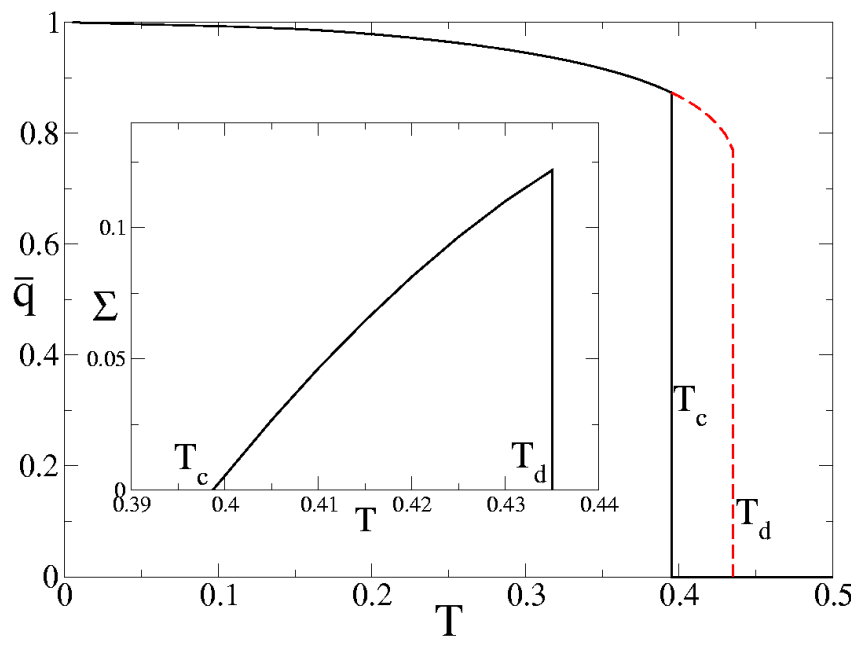

FIG. 1: The 1RSB overlap $\bar{q}$ as a function of the reduced temperature. The full line is the stable part of the curve, the dashed line is the metastable part. Inset: the complexity $\Sigma(T)$ as a function of the temperature. It jumps discontinuously at $T_{d}$ where the metastable solution first appears and vanishes at $T_{c}$ where the glass transition takes place.

where the average is on the measure that defines $Z(q)$. In the following, we will restrict ourselves to consider real values of $q_{a b}$, without taking explicitly into account the trivial rotational symmetry 29$]$.

The replica symmetric (RS) solution corresponds to $q_{a b} \equiv q$ and as usual it turns out that $q=0$ is the correct solution. The RS free energy is simply $f_{R S}=-\beta / 4$ as in the Ising $p$-spin glass. In this regime the $\varphi_{m}$ are uniformly distributed in $[0,2 \pi)$, independent and rapidly evolving, as originally considered in 21, 22].

In the one step replica symmetry breaking (1RSB) ansatz one divides the matrix $q_{a b}$ in $n / m$ blocks of side $m$ [16]. The elements in the off-diagonal blocks are set to 0 while in the diagonal blocks RS is assumed and $q_{a b}=q$. In this case, Eq. (11) becomes:

$$
\begin{aligned}
\beta \phi(m, q)= & -\left(\beta^{2} / 4\right)\left[1+3(1-m) q^{4}-4 q^{3}\right] \\
& -m^{-1} \log \int_{0}^{\infty} d z z e^{-\frac{z^{2}}{2}} I_{0}(\beta l z)^{m},
\end{aligned}
$$

where $I_{n}$ indicates the modified Bessel function of first kind and $l=\sqrt{2 q^{3}}$. The self-consistency equation that defines $q$ is

$$
q=\frac{\int_{0}^{\infty} d z z e^{-\frac{z^{2}}{2}} I_{0}^{m}(\beta l z) \frac{I_{1}(\beta l z)^{2}}{I_{0}(\beta l z)^{2}}}{\int_{0}^{\infty} d z z e^{-\frac{z^{2}}{2}} I_{0}^{m}(\beta l z)} .
$$

These expressions are identical to the 1RSB free energy for the $(p=4) p$-spin model, the only difference being the presence of the Bessel function instead of the hyperbolic cosine in the last integral. Starting from this expression one can repeat the analysis of [30] to derive the full phase space structure of the model at the 1RsB level.

In Fig. 1 the solution $\bar{q}$ of Eq. (14) for $m=1$ is reported as a function of $T$. At high temperature $\bar{q}=0$ 
and the RS solution is recovered. On lowering the temperature, a solution $\bar{q} \neq 0$ first appears at $T_{d}$ (dashed line). However, it becomes stable only below the thermodynamic glass transition temperature $T_{c}<T_{d}$ (full line), as in standard first-order transitions. For $T_{c}<T<T_{d}$ the phase space is disconnected in an exponential number $\mathcal{N}(T)=\exp N \Sigma(T)$ of states. The 1RsB complexity $\Sigma(T)$ is reported in the inset of Fig. 1. At $T=T_{d}$ a dynamical transition is expected to take place [17, 20].

The stability of the 1RSB solution can be studied (within the assumption that $q_{a b}$ is real). It turns out that the 1RSB solution is thermodynamically stable for all temperatures, so in this case no Gardner transition is present [30, 31, 32].

The presence of a dynamical phase transition at a given value of the random laser pump intensity implies different interesting physical phenomena. These could be experimentally investigated by studying (for example via heterodyne experiments) the self correlation function of a specific frequency $\left(\omega_{m}\right)$ component of the electric field in the cavity:

$$
C\left(t, \omega_{m}\right)=\omega_{m} A_{m}^{2}\left\langle\exp \left\{i\left[\varphi_{m}(t+\tau)-\varphi_{m}(\tau)\right]\right\}\right\rangle_{\tau}
$$

On approaching $T_{d}$ from above (i.e. on increasing the pump power), the dynamics of phase variable $\varphi_{m}(t)$ becomes slower and slower and $C\left(t, \omega_{m}\right)$ is expected to decay towards zero in longer and longer times. At the dynamical transition, the dynamics of the $\varphi$ 's becomes non-ergodic, they are no longer able to explore the whole phase space and the function $C\left(t, \omega_{m}\right)$ decays towards a plateau. In other words, the mode's phases $\varphi_{m}(t)$ beside from small oscillation- are locked to some "equilibrium" values ("random mode locking"). Due to the non-vanishing value of the complexity $\Sigma$ at $T_{d}$, however, an exponentially large number of such equilibrium posi- tions exist, so giving rise to many different time structure of the electric field in random lasers. On further increasing the pump power, the complexity $\Sigma$ decreases and the ideal glassy state is reached at $T=T_{c}$. Below this value the number of equilibrium states is not exponential in $N(\Sigma=0)$. The equilibrium states below $T_{d}$ are difficult to reach because the needed time scale diverges [17]: interesting phenomena as aging, memory effects, and history dependent responses are expected to take place for $T<T_{d}$.

All these non equilibrium phenomena are theoretically predicted and, to some extent experimentally verified 17] in material systems (structural glasses, spin glasses,..). The absence of conclusive experiments is mainly due to the long time needed to "equilibrate" real glasses below $T_{d}$, a time which is dictated by the elementary time of the atomic/molecular dynamics in condensed matter (ps time scale). Photon dynamics in cavities is at least three order of magnitude faster. This observation drives us to propose the random lasers in disordered media as a benchmark to test experimentally the outcome of those theories, as the replica symmetry breaking.

In conclusion, the multi-mode dynamics in a random laser cavity has been investigated by statistical physics techniques, and a one step replica symmetry breaking phase transition has been found. Our results emphasize two important points: i) the light propagation in nonlinear disordered media shows the same complex behavior of the dynamics of glassy systems (aging, memory, ...); ii) due to the faster photon dynamics with respect to the atomic one, it is possible to use the random lasers as systems for experimentally testing the replica symmetry breaking transitions.

We are pleased to thank T. Castellani, L. Leuzzi, and F. Ricci-Tersenghi for useful discussions.
[1] H. Haken, Synergetics (Springer-Verlag, Berlin, 1978).

[2] H. Risken, The Fokker-Planck Equation (SpringerVerlag, Berlin, 1989).

[3] F. T. Arecchi, S. Boccaletti, and P. L. Ramazza, Physics Reports 318, 83 (1999).

[4] B. Vodonos, R. Weill, A. Gordon, A. Bekker, V. Smulakovsky, O. Gat, and B. Fischer, Phys. Rev. Lett. 93, 153901 (2004).

[5] A. Gordon, B. Vodonos, V. Smulakovski, and B. Fischer, Opt. Express 11, 3418 (2003).

[6] A. Gordon and B. Fischer, Opt. Comm. 223, 151 (2003).

[7] A. Gordon and B. Fischer, Opt. Lett. 28, 1326 (2003).

[8] R. Weill, A. Rosen, A. Gordon, O. Gat, and B. Fischer, Phys. Rev. Lett. 95, 013903 (2005).

[9] V. S. Lethokov, Sov. Phys. JETP (1968).

[10] N. M. Lawandy, R. M. Balachandran, A. S. L. Gomes, and E. Sauvain, Nature 368, 436 (1994).

[11] D. S. Wiersma, M. P. Vanalbada, and A. Lagendijk, Nature 373, 203 (1995).

[12] H. Cao, Y. G. Zhao, S. T. Ho, E. W. Seelig, Q. H. Wang, and R. P. H. Chang, Phys. Rev. Lett. 82, 2278 (1999).

[13] D. S. Wiersma and S. Cavalieri, Nature (2001).

[14] L. Florescu and S. John, Phys. Rev. Lett. 93, 013602 (2004).

[15] R. C. Polson and Z. V. Vardeny, Appl. Phys. Lett. 85, 1289 (2004).

[16] M. Mézard, G. Parisi, and M. A. Virasoro, Spin glass theory and beyond (World Scientific, Singapore, 1987).

[17] L. Cugliandolo, Dynamics of glassy systems, in "Slow relaxation and nonequilibrium dynamics in condensed matter", Les Houches Session 77, J-L Barrat et al eds. (Springer-Verlag, Berlin, 2002).

[18] J. Kosterlitz and D. Thouless, J.Phys.C 5, L124 (1972).

[19] L. Angelani, L. Casetti, M. Pettini, G. Ruocco, and F. Zamponi, Europhys. Lett. 62, 775 (2003).

[20] T. Castellani and A. Cavagna, J. Stat. Mech. p. P05012 (2005), arXiv:cond-mat/0505032.

[21] J. Ducuing and N. Bloembergen, Phys. Rev. 133, A1493 (1964).

[22] I. C. L. O'Bryan and I. M. Sargent, Phys. Rev. A 8, 
3071 (1973).

[23] W. E. Lamb Jr, Phys. Rev. 134, A1429 (1964).

[24] H. A. Haus, Waves and Fields in Optoelectronics (Prentice-Hall, Englewood Cliffs, N. J., 1984).

[25] R. W. Boyd, Nonlinear Optics (Academic Press, New York, 2002), 2nd ed.

[26] N. Bloembergen and Y. R. Shen, Phys. Rev. 133, A37 (1964).

[27] C. W. Gardiner and P. Zoller, Quantum Noise (SpringerVerlag, Berlin, 2004), 3rd ed.
[28] A. Yariv, Quantum Electronics (Saunders College, San Diego, 1991).

[29] K. Binder and A. P. Young, Rev. Mod. Phys. 58, 801 (1986)

[30] A. Montanari and F. Ricci-Tersenghi, Eur. Phys. J. B. (2003).

[31] E. Gardner, Nucl. Phys. B 257, 747 (1985).

[32] A. Crisanti, L. Leuzzi, and T. Rizzo, Phys. Rev. B 71, 094202 (2005). 